\title{
Nonsmooth maps and the fast-slow dynamics of sleep-wake regulation: Part II
}

\author{
Anne C. Skeldon, Gianne Derks, and Victoria Booth \\ Department of Mathematics, University of Surrey, Guildford, Surrey, GU2 7XH, UK \\ a.skeldon@surrey.ac.uk \\ Department of Mathematics, University of Surrey, Guildford, Surrey, GU2 7XH, UK \\ g.derks@surrey.ac.uk \\ Departments of Mathematics \& Anesthesiology, University of Michigan, 530 Church Street, Ann Arbor, \\ MI 48109-1043, USA \\ vbooth@umich.edu
}

\begin{abstract}
In part I, the Two-Process model for sleep-wake regulation was discussed and it was shown that it could usefully be represented as a one-dimensional map with discontinuities. Here we discuss some recent, more physiological, models of sleep wake dynamics. We describe how their fast-slow structure means that one can expect them to inherit many of the dynamical features of the Two-Process model.
\end{abstract}

\section{Neuronal models of sleep-wake regulation}

Recent experimental findings have led to the idea that sleep-wake regulation occurs by a 'flip-flop' switch between sleep and wake states generated through mutual inhibition between populations of sleep and wake promoting neurons [9]. This has led to several mathematical models that extend the ideas of the Two-Process (TP) model to a more physiological setting: a recent review is given in [1]. These models consider the interactions between sleep- and wake-promoting neuronal populations that are modulated by the homeostatic sleep drive and the circadian rhythm of the TP model. They are described by systems of ordinary differential equations of the form

$$
\begin{aligned}
\tau_{i} \frac{d \mathbf{U}}{d t}+\mathbf{U} & =\mathbf{G}(\mathbf{U}, H, C(t)) \quad \mathbf{U} \in R^{n}, \\
\chi(\mathbf{U}) \frac{d H}{d t}+H & =G_{H}(\mathbf{U}) .
\end{aligned}
$$

Here the elements of $\mathbf{U}$ represent activity levels of the neuronal populations either in terms of average firing rate or average membrane potential and, in some cases, the concentrations of key neurotransmitters. The variable $H$ represents the homeostatic sleep pressure and $C(t)$ is a periodic circadian external drive. The vector-valued function $\mathbf{G}$ is sigmoidal in the population activity levels. The function $G_{H}$ is sigmoidal with respect to the action of wake promoting neurons or is taken to be a hard switch between two threshold functions. The function $\chi(\mathbf{U})$ is either constant or takes one of two values dependent on the activity state of the wake population.

Two examples of models of this type are the Phillips and Robinson model (PR model) $[\mathbf{7}]$ and the Booth and Diniz-Behn model (BDB model) $[\mathbf{3}, \mathbf{5}]$. The PR model includes two neuronal populations, one promoting sleep and one promoting wake. The BDB model distinguishes three neuronal groups, one for wake, one for rapid eye movement (REM) sleep and one for non-REM (nREM) sleep.

Research Perspectives CRM Barcelona, Spring 2016, vol. 9, in Trends in Mathematics Springer-Birkhuser, Basel 
An essential common feature in these neuronal models is that, since $\tau_{i}$ is much smaller than both $\chi(\mathbf{U})$ and the period of $C(t)$, there is a separation of time scales between the neuronal dynamics and the dynamics of the homeostatic and circadian rhythms. Hence there is a slow manifold $\mathbf{U}_{0}(H, C)$ that, to lowest order, is given by

$$
\mathbf{U}_{0}=\mathbf{G}\left(\mathbf{U}_{0}, H, C\right) .
$$

In the simplest setting, considering sleep as consisting of one state (nREM), the sigmoidal assumptions mean that equation (3) has one or three solutions parameterized by $H$ and $C$. Consequently, the slow manifold takes the form of a folded surface with saddle-node bifurcations characterizing the position of the folds, as shown in Figure 1(a) and (b) for the $\mathrm{PR}$ and the $\mathrm{BDB}$ models respectively $[\mathbf{4}, \mathbf{1 0}]$. In this figure, in the fast dynamics, the 'upper' wake state and the 'lower' sleep states are stable, while the intermediate steady-states are unstable. The slow dynamics of the homeostat $H$ given by equation (2)
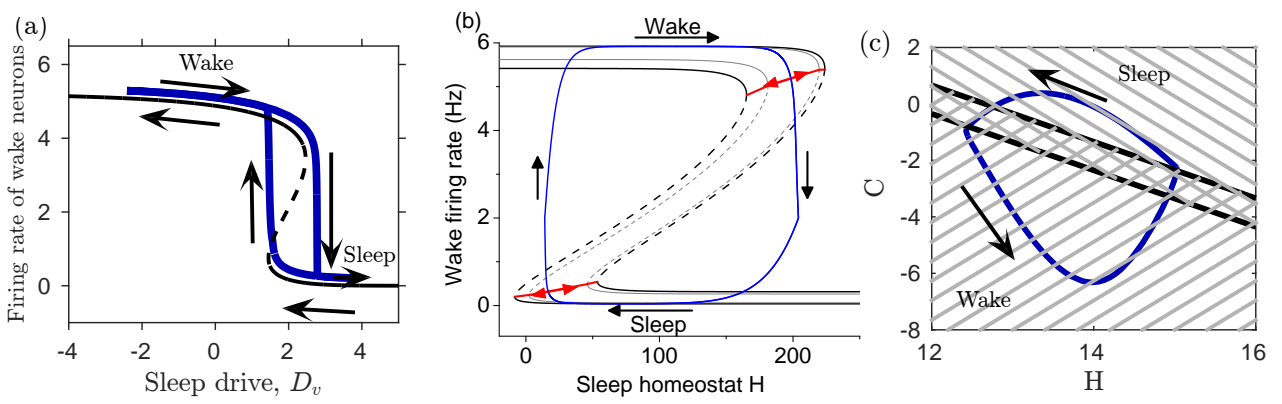

FiguRE 1. (a) The slow manifold of the PR model along with a typical trajectory. The trajectory has been offset from the slow manifold for clarity. (b) The slow manifold and a trajectory for the BDB model. (c) The trajectory shown in (a) re-plotted in the $(H, C)$-plane. The saddle-node bifurcations where switching between wake and sleep occur are represented by the thick black diagonal lines.

result in $H$ increasing (during wake) and decreasing (during sleep). Together with the periodic oscillation of the circadian $C$ this leads to relaxation oscillations between wake and sleep states, shown by the blue curves in Figure 1(a) and (b). In the PR model, Figure 1(a), the dynamics of $H$ and $C$ are combined in a single 'sleep' drive parameter, $D_{v}=H(t)-C(t)$. In the BDB model, $H$ and $C$ are represented as separate inputs to the neuronal populations, resulting in the slow manifold being most apparent as a function of $H$ with $C$ modulating the position of the slow manifold a function of time. An alternative illustration of these dynamics is to depict the slow manifold as a function of the two slow parameters, $H$ and $C$, as shown in Figure 1(c) where the slow manifold of the PR model has been projected onto the $(H, C)$-plane.

\section{Discussion}

Although the neuronal models and the TP model are formulated in different ways, they share many common features: in [6], it is shown how solutions of the PR model can be plotted in the form of the TP model and in $[\mathbf{1 0}]$ it is shown that the slow dynamics of the PR model and the TP model can be formally identified in the limit that the soft switching functions in the PR model are replaced by hard switches. Consequently, essentially the 
same kind of patterns of sleep and wake can be expected in the PR model as are seen in the TP model. Furthermore, many of the different behaviours seen in simulations of the PR model, such as those explaining different mammalian sleep patterns in [8], can then be understood as resulting from border collision bifurcations.

The common underlying structure described in equations (1) and (2), suggests that not only the PR model but other neuronal models can be related to the TP model and to one-dimensional maps with discontinuities. This is indeed the case for the BDB model, in its simplest setting, as shown in Figure 2. The sleep-wake switching behaviour of the BDB model can be plotted in the same spirit as the TP model (Figure 2(a)). Here, $H(t)$ switches between sleep and wake states and the position of the upper and lower saddlenode bifurcation points of the slow manifold are modulated by the circadian oscillation. The switch between sleep and wake states does not occur exactly when the trajectory reaches a threshold curve, as in the TP model, due to slow passage effects around the saddle-node points.

A one-dimensional map for successive sleep onset times can be computed for the BDB model (Figure 2(b)), where sleep onset timing is now specified relative to the phase of the circadian oscillation. This map is constructed by initialising the BDB model at a phase of the circadian cycle $\Phi_{0}^{n}$ at time $t=t_{0}^{n}$, with $C=C\left(t_{0}^{n}\right)$ and $H\left(t_{0}^{n}\right)$ such that $\left(H\left(t_{0}^{n}\right), C\left(t_{0}^{n}\right)\right)$ and the firing rate and neurotransmitter variables $\mathbf{U}$ are set at their values at the upper saddle-node point in Figure 1(b). The system is then evolved through one sleep-wake cycle until an upper saddle-node point is reached again at time $t=t_{0}^{n+1}$ and circadian phase $\Phi_{0}^{n+1}$. The map consists of all points $\Phi_{0}^{n+1} \bmod 1$ such that $\Phi_{0}^{n} \in[0,1]$. As in the TP model map, this map has gaps with infinite derivatives to the left of the gap and bounded derivatives to the right of the gap. These gaps occur due to model trajectories becoming tangent to the boundary curves defined by the saddle-node points of the slow manifold, as illustrated in terms of $H(t)$ in the lower panel of Figure 2(a). This reduction to a one-dimensional map is strongly suggestive that, like the PR model,
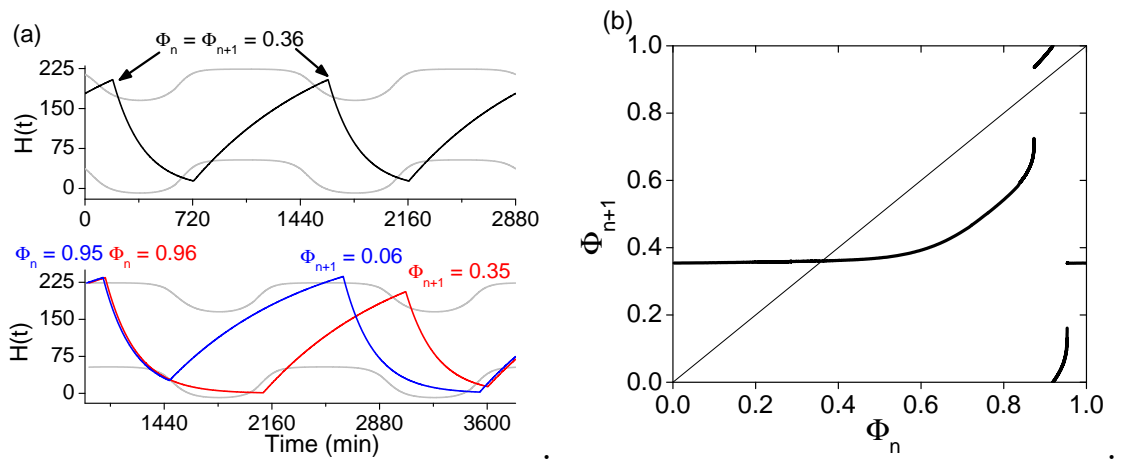

Figure 2. (a) Sleep-wake switching in the BDB model. The homeostatic sleep drive $H(t)$ varies between threshold curves determined by the circadian modulation of the $H$ values of the upper and lower saddle-node bifurcation points of the slow manifold (Figure 1(b)). Circadian phases of sleep onset times labeled for reference (colours match trajectory curves). (b) One-dimensional map of circadian phases of sleep onset $\Phi_{n+1}$ as a function of the previous sleep onset phase $\Phi_{n}$ computed from the BDB model when REM sleep is suppressed. 
border collisions are critical in understanding some of the dynamics of the BDB model.

Whereas the PR model only considers two neuronal populations and describes the two states of wake and sleep, the BDB model includes three neuronal groups and the three states of wake, nREM sleep and REM sleep. Humans normally oscillate between nREM and REM sleep during a typical night's sleep, with the longest interval of nREM sleep occurring at the beginning of sleep. By changing one of the parameters, the BDB model can capture the periodic switching between nREM and REM sleep. This transition occurs as a result of the steady-state of the lower branch of the slow manifold losing stability to be replaced by a stable periodic solution. The number of REM episodes during one night can be tuned, with Figures 1(b) and 2 illustrating the particular case where there are no REM episodes. In the more complicated dynamical regimes, where oscillations between REM and nREM sleep exist, the model can still be reduced to a one-dimensional map, but each additional REM episode results in an additional discontinuity in the map. This intriguing structure is currently under investigation [2].

There remain many unanswered questions and interesting dynamics to explore but a few points are clear. Sleep-wake regulation models are interesting and biologically relevant examples of non-smooth systems. Recognising their non-smooth nature is important both in gaining an in-depth understanding of their dynamical behaviour and in understanding the extent to which different models have different dynamics.

\section{Acknowledgements}

The authors thank the Centre de Recerca Matemàtica for the opportunity to work with each other and other participants of the intensive programme on Advances in Nonsmooth Dynamics. VB acknowledges her collaborator on this work Cecilia Diniz Behn and support from the National Science Foundation DMS-1412119.

\section{References}

[1] V. Booth and C.G. Diniz Behn. Physiologically-based modeling of sleep-wake regulatory networks. Mathematical Biosciences, 250:54-68, 2014.

[2] V. Booth, I. Xique, and C.G. Diniz Behn. A one-dimensional map for the circadian modulation of sleep in a human sleep-wake regulatory network model. Centre de Recerca Matemàtica, Preprint \#1223, 2016.

[3] C. Diniz Behn and V. Booth. Simulating microinjection experiments in a novel model of the rat sleep-wake regulatory network. J Neurophysiol, 103:1937-1953, 2010.

[4] B.D. Fulcher, A.J. Phillips, and P.A. Robinson. Modeling the impact of impulsive stimuli on sleepwake dynamics. Phys. Rev. E. Stat. Nonlin. Soft. Matter Phys., 78:051920, 2008.

[5] R. D. Gleit, C. Diniz Behn, and V. Booth. Modeling interindividual differences in spontaneous internal desynchrony patterns. Journal of Biological Rhythms, 28:339-355, 2013.

[6] A. J. Phillips and P. A. Robinson. Sleep deprivation in a quantitative physiologically based model of the ascending arousal system. J. Theor. Biol., 255:413-423, 2008.

[7] A.J. Phillips and P.A. Robinson. A quantitative model of sleep-wake dynamics based on the physiology of the brainstem ascending arousal system. J. Biol. Rhythms, 22:167-179, 2007.

[8] A.J. Phillips, P.A. Robinson, D.J. Kedziora, and R.G. Abeysuriya. Mammalian sleep dynamics: how diverse features arise from a common physiological framework. PLoS. Comput. Biol., 6:e1000826, 2010b.

[9] C.B. Saper, T.E. Scammell, and J. Lu. Hypothalamic regulation of sleep and circadian rhythms. Nature, 437:1257-1263, 2005.

[10] A.C. Skeldon, D.-J. Dijk, and G. Derks. Mathematical models for sleep-wake dynamics: comparison of the two-process model and a mutual inhibition neuronal model. PLoS ONE, 10:e103877, 2014. 